\title{
Application of optical switch based on the liquid-crystal modulated self-collimating photonic crystals
}

\author{
Xiaohui $\mathrm{Li}^{1, \mathrm{a}^{*}}$, Huiyu Yang ${ }^{2, b}$ \\ ${ }^{1}$ College of electronic and information Engineering, Chongqing Radio And TV University, \\ No.1,Hualong Road, Jiulong District, Chongqing, China \\ ${ }^{2}$ CenterLab of Measurement \& Physical Test and Chemical Analysis in Chongqing Jianshe \\ Industry,Ltd.No.10,Xiejiawang Road, Jiulong District, Chongqing, China \\ a651739024@qq.com, ${ }^{\mathrm{b}} 461956904 @ q q . c o m$
}

\begin{abstract}
Keywords: photonic crystals; optical switch; self-collimation; liquid crystals
Abstract. The tunability on the refractive index of the liquid crystals makes the photonic crystal optical switch possible, which comprises liquid crystal materials. In this article, based on the light induced alignment of liquid crystals and the self-collimation effect in the photonic crystals, an all-optical switch is proposed. The photonic crystal consists of silicon rods arranged in a triangular lattice in a liquid crystal background. The plane wave expansion (PWE) method is used to calculate the equi-frequency contours(EFCs) and the band diagrams. The transmission spectra are obtained by using the finite-difference time-domain (FDTD) method. The propagation of light in such structure is simulated with the FDTD method.
\end{abstract}

\section{Introduction}

Photonic crystal is a novel artificial materials that consists of periodic medium array. Satellite is the important communication platform in space optical communication, but the payload of rocket and satellite is very small. To effectively reduce the launch cost so as to increase the efficiency per weight unit, the overall weight of satellite has to be strictly controlled. Optical antenna, as an essential part of wireless laser communication system, has been developed toward integration and miniaturization which is of great importance.

The miniaturization and integration of optical antenna can be implemented by improving optical path, reducing optical component, introducing novel optical devices. The extensively used novel optical devices such as photonic crystal materials ${ }^{[1][2]}$, binary optical elements, have increasing significance in miniaturization and integration of optical devices, and have evoked great research interest over the world.

Two dimensional photonic crystal waveguide has outstanding light guide performance. In 1996, Mekis ${ }^{[3]}$ proposed photonic crystal right angle waveguide, which makes the photonic crystal waveguide more flexible to different applications. Traditional medium waveguide has radiation loss when it pass through a right angle waveguide curve, which result in low transmission efficiency of $30 \%$ only. Therefore, the integration of optical devices is impossible to be realized scale-wise using traditional medium waveguide. On the contrary, the bend loss of photonic crystal waveguide is so small that it is possible to integrate photonic crystal devices in millimeter scale. In traditional optical antenna, a large number of lens and reflectors are required to control optical path. If the integrated optical devices based on the photonic crystal waveguide are used, a certain number of lens and reflectors can be replaced so as to achieve the miniaturization of optical antenna.

\section{Optical switch structure of photonic crystal}

Photonic crystal is a novel artificial structure functional materials that consists of periodic medium array. In 1991, Yablonovitch fabricated the first omni-directional photonic crystal band gap structure ${ }^{[5],}$ and verified the existence of photonic forbidden bands. If the photon energy lies in the frequency range of forbidden band, then it cannot be transmitted in the medium. If line defect is introduce in the 
photonic crystal, it forms photonic crystal waveguide which behave like optical waveguide where the light within photonic crystal forbidden band can be transmitted with low energy loss. When the line defect bend 90 degree, the light can also be transmitted with extremely low energy loss, the theoretical transmission efficiency can reach $100 \%$. Take advantage of this property, photonic crystal waveguide can be used for optical path integration and optical interconnection ${ }^{[4][5][6]}$.

In space optical communication, the beacon optical waveband is usually $0.800 \sim 0.860 \mu \mathrm{m}$, signal optical waveband is $0.980^{\mu m}, 1.550 \mu \mathrm{m}$. Take $1.550^{\mu \mathrm{m}}$ for example, we designed the photonic crystal waveguide for the TM mode in this waveband, which is used to control the transmission light path in optical antenna. We also conduct FDTD simulation on the light transmission in right angle waveguide.

We use PWE method for optimal design for $1.550 \mu m$ waveband, where light within this waveband lies in the photonic forbidden band frequency range. The software used is the open source software MIT Photonic Bands(MPB) ${ }^{[7]}$. Figure 1 shows the photonic crystal structure after optimal design. The photonic crystal is composed of 21 by 21 uniform medium cylinder in the air with infinite length, whose dielectric constant is $\varepsilon=11.56$, distance between cylinders is $a=0.550^{\mu m}$. In the rest of paper, a is considered as normalization unit in calculation. The normalized wave length corresponding to $1.550^{\mu m}$ is 2.818 , normalized frequency is 0.355 . The right angle photonic crystal can be obtained by removing part of the medium cylinder.

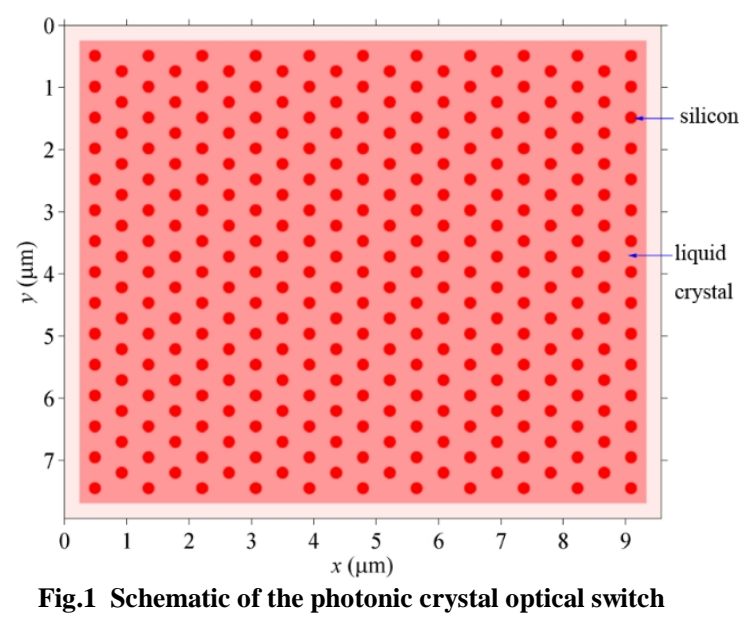

Figure 2 shows the band structure of the photonic crystal, from which it can be observed that there are three band gaps. The first gap is centered at 0.355 , band gap ranges from 0.2876 to 0.4228 , band gap width is $38.08 \%$ which is very large. The light that lies in this forbidden band of the photonic crystal, it can only transmit along photonic crystal waveguide and the energy also primarily transmit in waveguide. On the other hand, the light outside forbidden range scatters severely, no matter it is in the air or in photonic crystal, thus diverges rapidly.

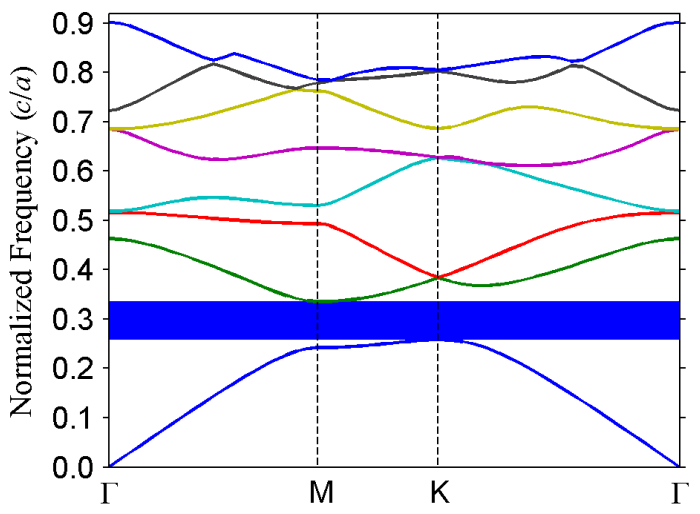

Fig.2 The TM mode band structure of the photonic crystal 


\section{FDTD simulation optical wave transmission in photonic crystal waveguide}

In order to test optical transmission in photonic crystal waveguide, we used FDTD Method to simulate optical transmission. The simulation software used is MIT Electromagnetic Equation Propagation(Meep ${ }^{[8]}$.

The light source used is a Gaussian light source whose normalized frequency of is 0.355 and frequency band is 0.05 . The light source input from the waveguide entrance right below the photonic crystal and output from exit on the right. Figure 3 shows the simulation on filed distribution when light wave is transmitting in right angle photonic crystal waveguide. It can be seen from the figure that the transmission of light wave energy concentrates in right angle photonic waveguide, the energy loss during transmission is extremely low.

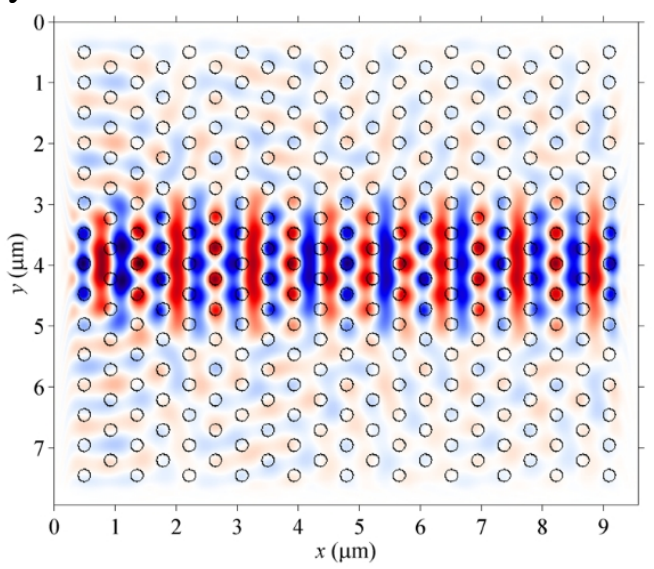

Fig.3 FDTD Simulations of the light propagation in the photonic crystal optical switch

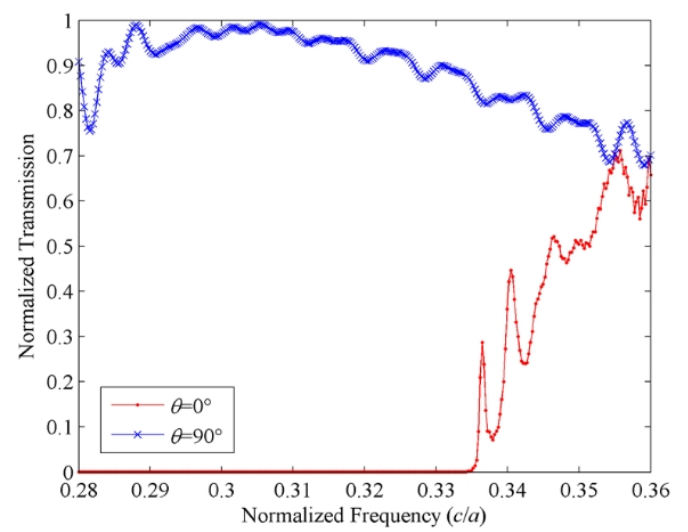

Fig.4. Distribution of simulated wave field at the right angle spread of photonic crystals

\section{Conclusion}

This paper proposes to introduce photonic crystal integrated optical path in optical antenna to replace traditional optical lens so as to control light transmission for the purpose of miniaturizing optical antenna. We designed photonic crystal waveguide structure for the wave band of $1.550^{\mu \mathrm{m}}$ such that the light wave that lies in a wide frequency band around this wave band can be transmitted in right angle photonic crystal waveguide with low energy loss. Finally, the FDTD method is used to simulate optical wave transmission. This paper is of theoretical significance in the research of photonic crystal application in miniaturization of optical antenna.

\section{Acknowledgements}

This research supported by the project "Research on negative refraction photonic crystal and its application" in Commission of Education of Chongqing,China(Grant No.KJ 131604). 


\section{References}

[1] Jianling Yin, Xuguang Huang, Songhao Liu. Photonic Crystal Field-Sensitive Polarizer and Switch Modulated by Bemaic Liquid Crystals[J].Acta Physica Sinica,2006,55(10): 5268-5276.

[2] Bo Yang , Jingqiu Liang, Zhongzhu Liang. The Optical Properties of A Liquid Crystal-Metal Photonic Crystal Waveguide [J]. Chinese Journal of Luminescence,2011,32(11): 1159-1164.

[3] Heming Chen,Guodong Wang. Design of New Photonic Crystal All-Optical Switching Based on Dynamical Shift of Defect Mode [J]. Acta Optica Sinica,2011 (03): 267-272.

[4] Kui Han, Ziyu Wang, Xiaopeng, Shen. Mach-Zehnder Interferometer Designed Based on Self-Collimating Beams and Photonic Band Gap in Photonic Crystals [J].Acta Physica Sinica,2011(04): 277-283.

[5] Shengyu Chen, Dongxia Zhuang, Zexuan Qiang. 1X4 Optical Multiplexer Based on Self-Collimation Effect in Silicon Photonic Crystals [J]. Optics and Precision Engineering, 2012,20 (12): 2626-2632.

[6] Lei Li, Guiqiang Liu, Yuanhao Chen. An Optical Switch Based on Coupled Heterostructure Photonic-Crystal Waveguide [J]. Acta Optica Sinica,2013 (01): 216-222.

[7] Heming Chen,Xiaoying Wei. The Design of High-speed Photonic Crystal Optical Switch [J]. Opto-Electronic Engineering,2013(11): 34-39.

[8] Jihuan Pan,An Su,Chengju Meng. Modulation of Optical Thickness on Transmission Spectrum of Photonic Crystal [J]. Laser \& Infrared,2014(05): 559-562. 